SLAC-PUB-8505

July 2000

\title{
A STUDY OF THE STRUCTURE OF $\mathrm{e}^{+} \mathrm{e}^{-} \rightarrow b \bar{b} g$ EVENTS AND IMPROVED LIMITS ON THE ANOMALOUS CHROMOMAGNETIC COUPLING OF THE $b$-QUARK ${ }^{*}$
}

\author{
The SLD Collaboration ${ }^{* *}$ \\ Stanford Linear Accelerator Center \\ Stanford UniversityГStanfordГCА 94309
}

\begin{abstract}
The structure of $\mathrm{e}^{+} \mathrm{e}^{-} \rightarrow b \bar{b} g$ events was studied using $Z^{0}$ decays recorded in the SLD experiment at SLAC. Three-jet final states were selected and the CCD-based vertex detector was used to identify two of the jets as $b$ or $\bar{b}$. Distributions of the gluon energy and polar angle were measured over the full kinematic range $\Gamma$ and compared with perturbative QCD predictions. The energy distribution is potentially sensitive to an anomalous $b$ chromomagnetic moment $\kappa$. We measured $\kappa$ to be consistent with zero and set limits on its value $\Gamma-0.11<\kappa<0.08$ at $95 \%$ c.l. (preliminary).
\end{abstract}

Contributed to the $X X X^{\text {th }}$ International Conference on High Energy Physics, July 27-August 2, 2000, Osaka, Japan; Ref: Pa03-691.

* Work supported by Department of Energy contract DE-AC03-76SF00515 (SLAC). 


\section{Introduction}

The observation of $e^{+} e^{-}$annihilation into final states containing three hadronic jets $\Gamma$ and their interpretation in terms of the process $e^{+} e^{-} \rightarrow q \bar{q} g[1] \Gamma$ provided the first direct evidence for the existence of the gluon $\Gamma$ the gauge boson of the theory of strong interactions $\Gamma$ Quantum Chromodynamics (QCD). In subsequent studies the jets were usually energy ordered $\Gamma$ and the lowest-energy jet was assigned as the gluon; this is correct roughly $80 \%$ of the time $\Gamma$ but preferentially selects low-energy gluons. If the gluon jet could be tagged explicitlyTevent-by-event $\Gamma$ the full kinematic range of gluon energies could be explored $\Gamma$ and more detailed tests of QCD could be performed [2]. Due to advances in vertex-detection this is now possible using $\mathrm{e}^{+} \mathrm{e}^{-} \rightarrow b \bar{b} g$ events. The large mass and relatively long lifetime $\Gamma \sim 1.5$ ps $\Gamma$ of the leading $B$ hadron in $b$-quark jets [3] lead to decay signatures which distinguish them from lighter-quark $(u \Gamma d \Gamma s$ or c) and gluon jets. We used our original (1992-5) and upgraded (1996-8) CCD vertex detectors $[4 \Gamma 5]$ to identify in each event the two jets that contain the $B$ hadrons $\Gamma$ and hence to tag the gluon jet. This allowed us to measure the gluon energy and polar-angle distributions over the full kinematic range.

Additional motivation to study the $b \bar{b} g$ system has been provided by measurements involving inclusive $Z^{0} \rightarrow b \bar{b}$ decays. Several reported determinations $[6]$ of $R_{b}=$ $\Gamma\left(Z^{0} \rightarrow b \bar{b}\right) / \Gamma\left(Z^{0} \rightarrow q \bar{q}\right)$ and the $Z^{0}-b$ parity-violating coupling parameter $\Gamma A_{b} \Gamma$ differed from Standard Model (SM) expectations at the few standard deviation level. Since

one expects new high-mass-scale dynamics to couple to the massive third-generation fermions $\Gamma$ these measurements aroused considerable interest and speculation. We have therefore investigated in detail the strong-interaction dynamics of the $b$-quark. We have compared the strong coupling of the gluon to $b$-quarks with that to light- and charm-quarks [7] Tas well as tested parity $(\mathrm{P})$ and charge $\oplus$ parity $(\mathrm{CP})$ conservation at the $b \bar{b} g$ vertex [8]. We have also studied the structure of $b \bar{b} g$ events via the distributions of the gluon energy and polar angle with respect to (w.r.t.) the beamline [9]; here we present a preliminary update of these measurements using a data sample more than 3 times larger than in our earlier study. We compare these results with perturbative QCD predictionsTincluding a recent calculation at next-to-leading order (NLO) which takes quark mass effects into account [10].

In QCD the chromomagnetic moment of the $b$ quark is induced at the one-loop level 
and is of order $\alpha_{s} / \pi$. A more general $b \bar{b} g$ Lagrangian term with a modified coupling [11] may be written:

$$
\mathcal{L}^{b \bar{b} g}=g_{s} \bar{b} T_{a}\left\{\gamma_{\mu}+\frac{i \sigma_{\mu \nu} k^{\nu}}{2 m_{b}}\left(\kappa-i \tilde{\kappa} \gamma_{5}\right)\right\} b G_{a}^{\mu}
$$

where $\kappa$ and $\tilde{\kappa}$ parameterize the anomalous chromomagnetic and chromoelectric momentsTrespectively Wwich might arise from physics beyond the SM. The effects of the chromoelectric moment are sub-leading w.r.t. those of the chromomagentic moment $\Gamma$ so for convenience we set $\tilde{\kappa}$ to zero. A non-zero $\kappa$ would modify [11] the gluon energy distribution in $b \bar{b} g$ events relative to the standard QCD case. Hence we have used our larger data sample to set improved limits on $\kappa$.

\section{$2 \quad b \bar{b} g$ Event Selection}

We used hadronic decays of $Z^{0}$ bosons produced by $e^{+} e^{-}$annihilations at the SLAC Linear Collider (SLC) which were recorded in the SLC Large Detector (SLD) [12]. The criteria for selecting $Z^{0}$ decays $\Gamma$ and the charged tracks used for flavor-tagging $\Gamma$ are described in [7Г13]. We applied the JADE algorithm [14] to define jets Tusing a scaledinvariant-mass criterion $y_{c u t}=0.02$. Events classified as 3 -jet states were retained if all three jets were well contained within the barrel tracking system $\Gamma$ with polar angle $\left|\cos \theta_{\text {jet }}\right| \leq 0.71$. From our 1993-98 data samplesTcomprising roughly 5001000 hadronic $Z^{0}$ decays $\Gamma 1261871$ events were selected. In order to improve the energy resolution the jet energies were rescaled kinematically according to the angles between the jet axes $\Gamma$ assuming energy and momentum conservation and massless kinematics. The jets were then labelled in order of energy such that $E_{1}>E_{2}>E_{3}$.

Charged tracks with a large transverse impact parameter w.r.t. the measured interaction point (IP) were used to $\operatorname{tag} b \bar{b} g$ events [7]. The resolution on the impact parameterTprojected in the plane normal to the beamline $\Gamma d \Gamma i s \sigma_{d}=11 \oplus 70 /\left(p_{\perp} \sqrt{\sin \theta}\right)$ (1993-5) and $8 \oplus 33 /\left(p_{\perp} \sqrt{\sin \theta}\right)$ (1996-8) $\mu \mathrm{m} \Gamma$ where $p_{\perp}$ is the track transverse momentum in $\mathrm{GeV} / \mathrm{c} \Gamma$ and $\theta$ the polar angle $\Gamma$ w.r.t. the beamline. The jet flavor tag was based on the number of tracks per jet $\Gamma N_{s i g}^{\text {jet }} \Gamma$ with $d / \sigma_{d} \geq 3[9]$. Events were retained in which exactly two jets were $b$-tagged by requiring each to have $N_{\text {sig }}^{\text {jet }} \geq 2$ Tand in which the remaining jet had $N_{s i g}^{\text {jet }}<2$ and was hence tagged as the gluon; 8196 events were selected. The efficiency for selecting true $b \bar{b} g$ events is $12 \%$. This was estimated using a 


\begin{tabular}{|c|c|c|}
\hline Jet label & \# Tagged gluon jets & Purity \\
\hline 3 & 1140 & $94.4 \%$ \\
2 & 155 & $90.1 \%$ \\
1 & 34 & $73.1 \%$ \\
\hline
\end{tabular}

Table 1: Estimated purities of the tagged gluon-jet samples.

simulated event sample generated with JETSET 7.4 [15] Twith parameter values tuned to hadronic $e^{+} e^{-}$annihilation data [16] $\Gamma$ combined with a simulation of $B$-decays tuned to $\Upsilon(4 \mathrm{~S})$ data [17] and a simulation of the detector. The efficiency peaks at about $15 \%$ for $15 \mathrm{GeV}$ gluons. Lower-energy gluon jets are sometimes merged with the parent $b$-jet by the jet-finder. At higher gluon energies the the correspondingly lower-energy $b$-jets are harder to tag $\Gamma$ and there is also a higher probability of losing a jet outside the detector acceptance.

For the selected event sample $\Gamma$ Fig. 1 shows the $N_{s i g}^{j e t}$ distributions separately for jets $1 \Gamma 2$ and 3 . In about $16 \%$ of cases the gluon-tagged jet is not the lowest-energy jet (jet 3). The simulated contributions from true gluons are indicated [18] and the estimated gluon purities [18] are listed in Table 1. The inclusive gluon purity of the tagged-jet sample is $93 \%$. With this sample we formed the distributions of two gluonjet observables $\Gamma$ the scaled energy $x_{g}=2 E_{\text {gluon }} / \sqrt{s} \Gamma$ and the polar angle w.r.t. the beamline $\Gamma \theta_{g}$. The distributions are shown in Fig. 2. The simulation is also shown; it reproduces the data.

The backgrounds were estimated using the simulation and are of three types: non- $b \bar{b}$ events; $b \bar{b}$ but non- $b \bar{b} g$ events; and mis-tagged events. These are shown in Fig. 2. The non- $b \bar{b}$ events ( $\sim 5 \%$ of the selected sample) are mainly $c \bar{c} g$ events $\Gamma 92 \%$ of which had the gluon jet correctly tagged. There is a small contribution $(\sim 0.1 \%$ of the sample) from light-quark events. The dominant background is formed by $b \bar{b}$ but non- $b \bar{b} g$ events. These are true $b \bar{b}$ events that were not classified as 3 -jet events at the parton levelГbut were poorly reconstructed and tagged as 3 -jet $b \bar{b} g$ events in the detector using the same jet algorithm and $y_{c u t}$ value. This arises from the broadening of the particle flow around the original $b$ and $\bar{b}$ directions due to hadronization $\Gamma$ especially the relatively hightransverse-momentum $B$-decay products $\Gamma$ which causes the jet-finder to reconstruct a 


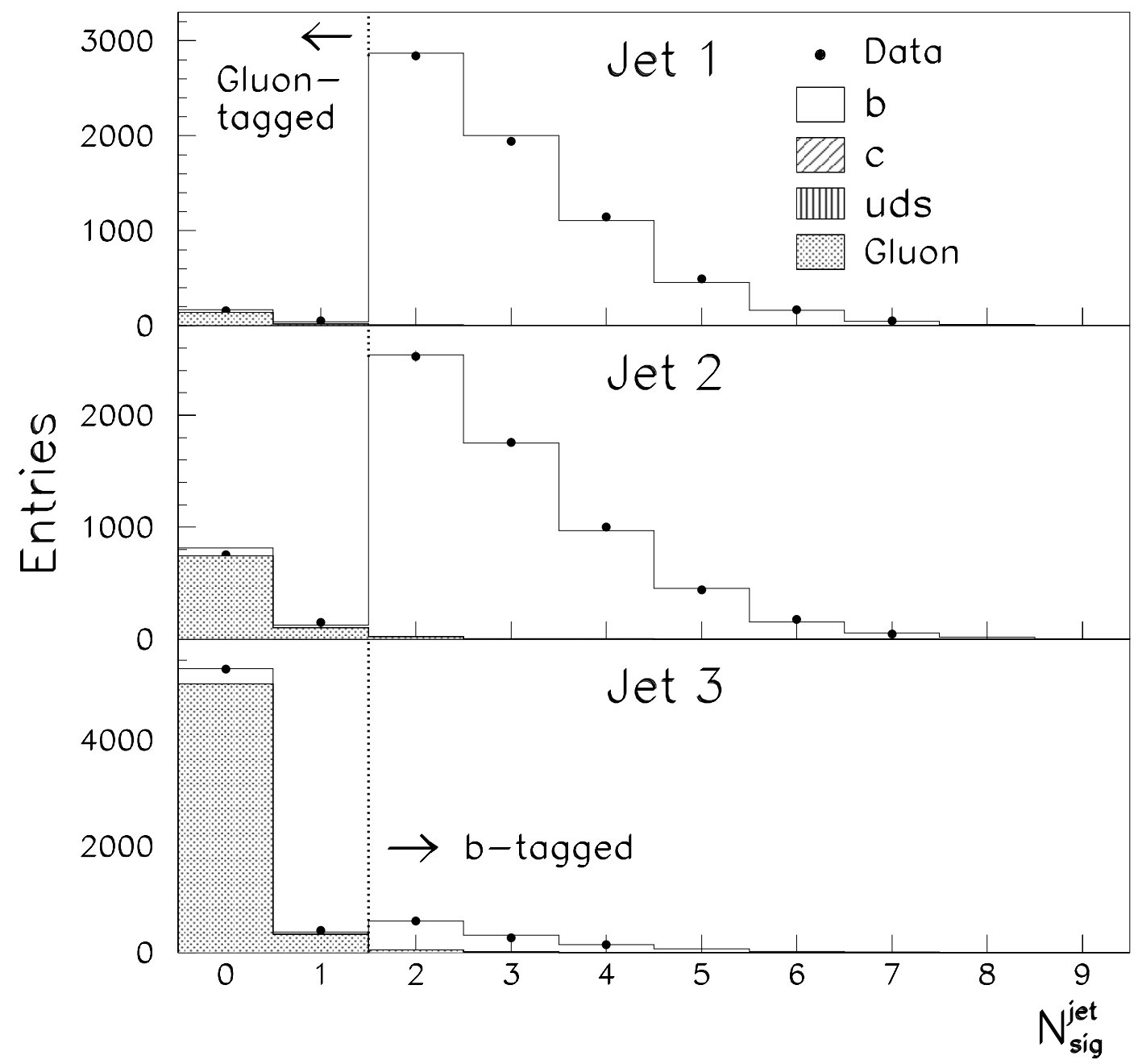

Figure 1: The $N_{s i g}^{\text {jet }}$ distributions for jets in $b \bar{b} g$-tagged eventsTlabelled according to jet energy (dots); errors are statistical. Histograms: simulated distributions showing jet flavor contributions. 

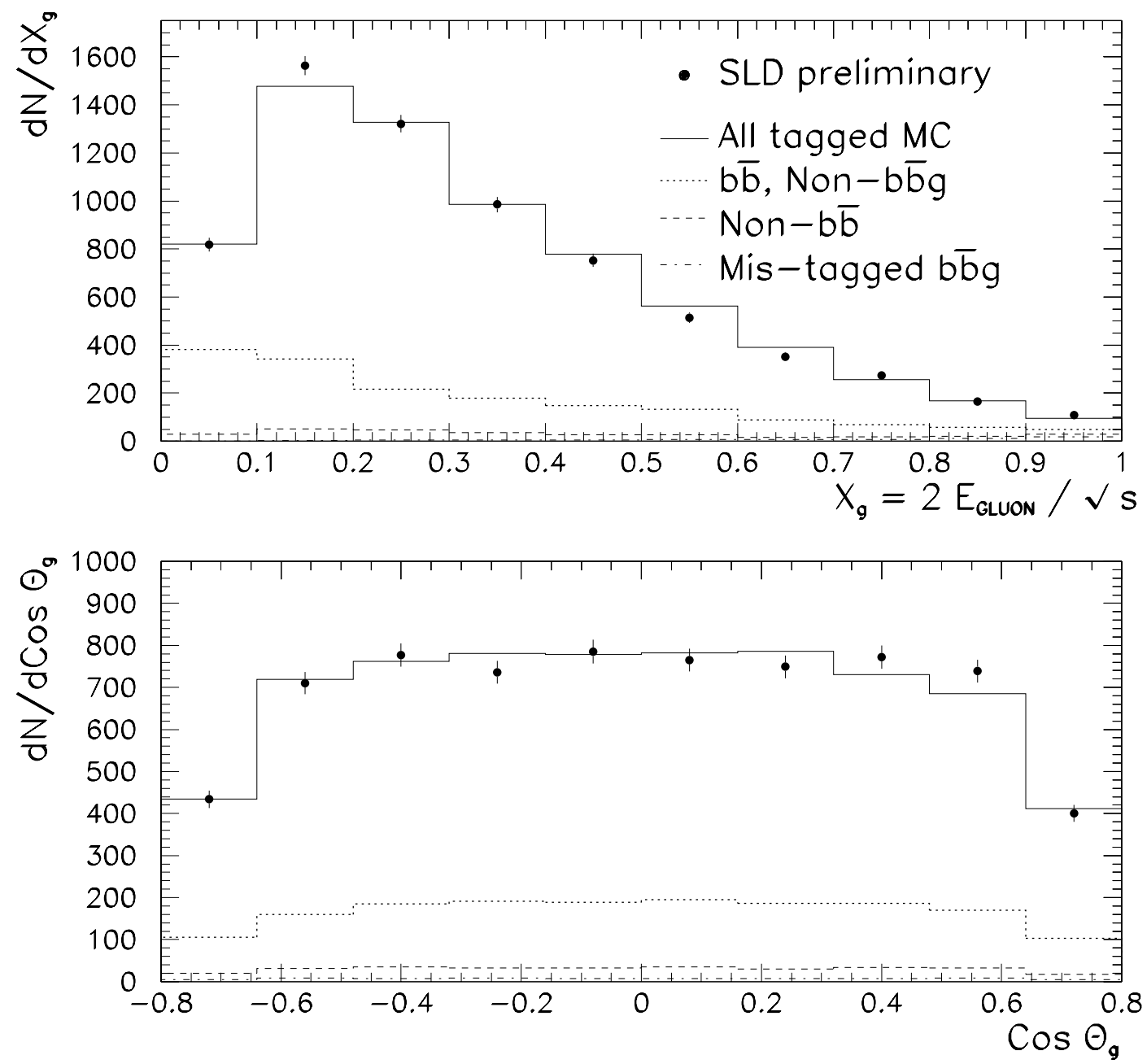

Figure 2: Raw measured distributions of (a) $x_{g}$ and (b) $\cos \theta_{g}$ (dots); errors are statistical. Histograms: simulated distributions including background contributions. 
'fake' third jetTalmost always assigned as the gluon. The population of such fake gluon jets peaks at low energy (Fig. 2(a)). Mis-tagged events $\Gamma$ in which the gluon jet was mis-tagged as a $b / \bar{b}$-jet and the $b$ or $\bar{b}$ jet enters into the measured distributions $\Gamma$ comprise less than $1 \%$ of the total sampleTbut make a substantial contribution in the highest $x_{g}$ bin.

The distributions were corrected to obtain the true gluon distributions $D^{\text {true }}(X)$ by applying a bin-by-bin procedure: $D^{\text {true }}(X)=C(X)\left(D^{\text {raw }}(X)-B(X)\right)$ Twhere $X=x_{g}$ or $\cos \theta_{g} \Gamma D^{\text {raw }}(X)$ is the raw distribution $\Gamma B(X)$ is the background contribution $\Gamma$ and $C(X) \equiv D_{M C}^{t r u e}(X) / D_{M C}^{r e c o n}(X)$ is a correction that accounts for the efficiency for accepting true $b \bar{b} g$ events into the tagged sampleTas well as for bin-to-bin migrations caused by hadronization $\Gamma$ the resolution of the detectorTand bias of the jet-tagging technique. Here $D_{M C}^{t r u e}(X)$ is the true distribution for MC-generated $b \bar{b} g$ events $\Gamma$ and $D_{M C}^{r e c o n}(X)$ is the resulting distribution after full simulation of the detector and application of the same analysis procedure as applied to the data.

As a cross-check Tan alternative correction procedure was employed in which binto-bin migrations $\Gamma$ which can be as large as $20 \% \Gamma$ were explicitly taken into account: $D^{\text {true }}\left(X_{i}\right)=M\left(X_{i}, X_{j}\right)\left(D^{\text {raw }}\left(X_{j}\right)-B\left(X_{j}\right)\right) / \epsilon\left(X_{i}\right)$ Twith the unfolding matrix $M\left(X_{i}, X_{j}\right)$ defined by $D_{M C}^{\text {true }}\left(X_{i}\right)=M\left(X_{i}, X_{j}\right) D_{M C}^{\text {recon }}\left(X_{j}\right)$ Twhere true $b \bar{b} g$ events generated in bin $i$ may $\Gamma$ after reconstruction $\Gamma$ be accepted into the tagged sample in bin $j . \epsilon(X)$ is the efficiency for accepting $b \bar{b} g$ events in bin $i$ into the tagged sample. The resulting distributions of $x_{g}$ and $\cos \theta_{g}$ are statistically indistinguishable from the respective distributions yielded by the bin-by-bin method.

The fully-corrected distributions are shown in Fig. 3. Since $\Gamma i n$ an earlier study $[7] \Gamma$ we verified that the overall rate of $b \bar{b} g$-event production is consistent with QCD expectations $\Gamma$ we normalised the gluon distributions to unit area and we study further the distribution shapes. The $x_{g}$ distribution rises $\Gamma$ peaks around $x_{g} \sim 0.15 \Gamma$ and decreases towards zero as $x_{g} \rightarrow 1$. The peak is a kinematic artifact of the jet algorithm $\Gamma$ which ensures that gluon jets are reconstructed with a non-zero energy which depends on the $y_{c}$ value. The $\cos \theta_{g}$ distribution is flat.

We have considered sources of systematic uncertainty that potentially affect our results. These may be divided into uncertainties in modelling the detector and uncertainties in the underlying physics modelling. To estimate the first case we systematically varied the track and event selection requirements $\Gamma$ as well as the tracking 

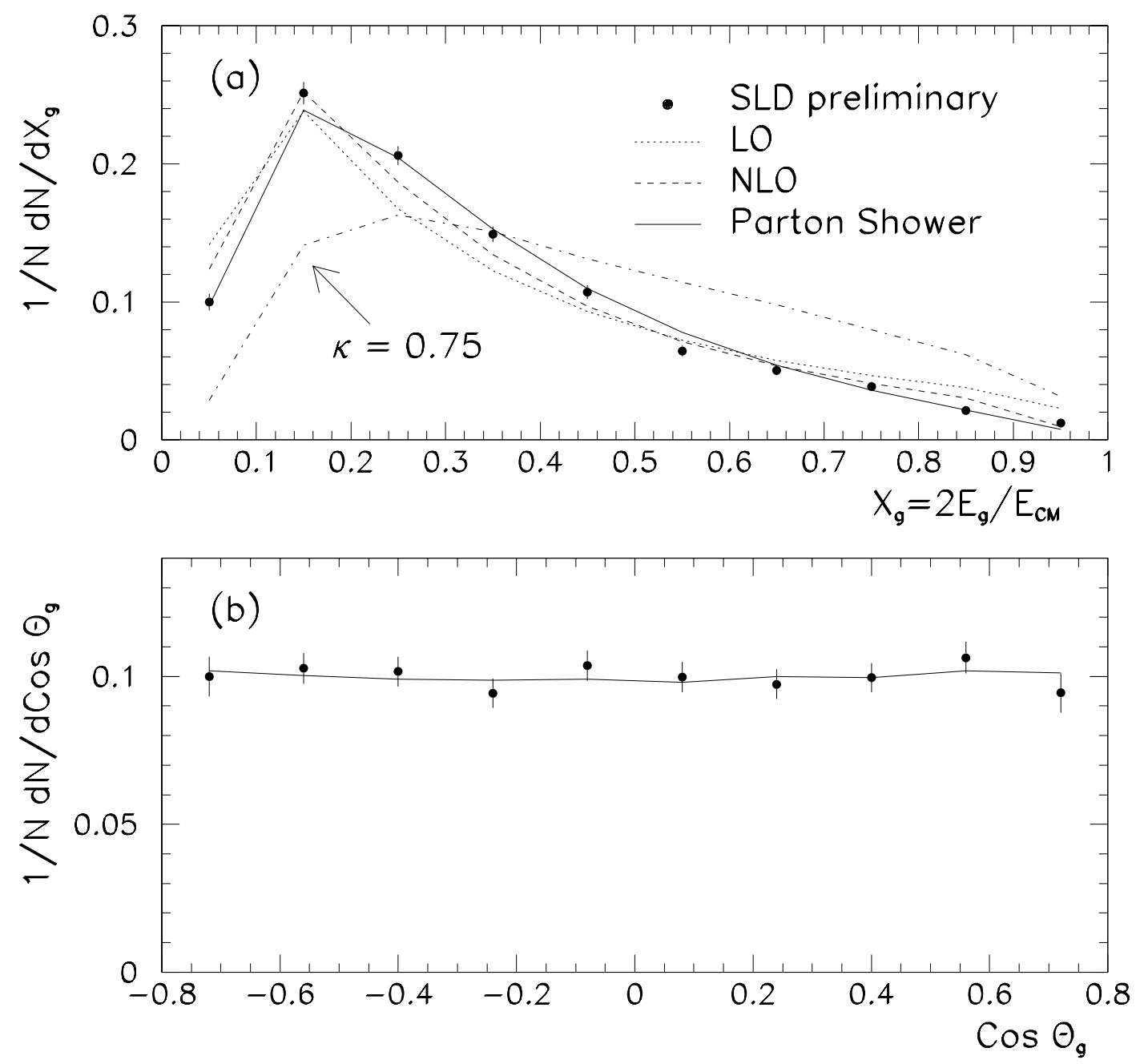

Figure 3: Corrected distributions of (a) $x_{g}$ and (b) $\cos \theta_{g}$ (dots); errors are statistical. Perturbative QCD predictions (see text) are shown as lines joining entries plotted at the respective bin centers. 
efficiency [7Г13]. In the second case parameters used in our simulationTrelating mainly to the production and decay of charm and bottom hadrons $\Gamma$ were varied within their measurement errors [13]. For each variation the data were recorrected to derive new $x_{g}$ and $\cos \theta_{g}$ distributions $\Gamma$ and the deviation w.r.t. the standard case was assigned as a systematic uncertainty. None of the variations affects our conclusions. All uncertainties were conservatively assumed to be uncorrelated and were added in quadrature in each bin of $x_{g}$ and $\cos \theta_{g}$. Since we are considering only the shapes of the distributions $\Gamma$ the systemtic errors are much smaller than the statistical errorsTexcept in the highest $x_{g}$ bin.

\section{Comparison with QCD Predictions}

We compared the data with perturbative QCD predictions for the same jet algorithm and $y_{c}$ value. We used leading-order (LO) and NLO results based on recent calculations [10] in which quark mass effects were explicitly taken into account; a $b$-mass value of $m_{b}\left(m_{Z}\right)=3 \mathrm{GeV} / c^{2}$ was used [19]. We also derived these distributions using the 'parton shower' (PS) implemented in JETSET. This is equivalent to a calculation in which all leading $\Gamma$ and a subset of next-to-leading $\Gamma \ln y_{c}$ terms are resummed to all orders in $\alpha_{s}$. In physical terms this allows events to be generated with multiple orders of parton radiation $\Gamma$ in contrast to the maximum number of 3 (4) partons allowed in the LO (NLO) calculations Trespectively. Configurations with $\geq 3$ partons are relevant to the observables considered here since they may be resolved as 3 -jet events by the jet-finding algorithm.

These predictions are shown in Fig. 3. The three calculations are indistinguishable for the $\cos \theta_{g}$ distribution and reproduce the measured distribution $\Gamma$ which is almost flat in contrast to the $1+\cos ^{2} \theta_{q}$ distribution for quark jets. For $x_{g}$ Talthough the LO calculation reproduces the main features of the shape of the distribution $\Gamma$ it yields too few events in the region $0.2<x_{g}<0.5$ Tand too many events for $x_{g}<0.1$ and $x_{g}>0.5$. The NLO calculation is noticeably better $\Gamma$ but also shows a deficit for $0.2<x_{g}<0.4$. The PS calculation describes the data across the full $x_{g}$ range. These results suggest that multiple orders of parton radiation need to be included $\Gamma$ in agreement with our earlier measurements of jet energy distributions using flavor-inclusive $Z^{0}$ decays [20]. We also investigated LO and NLO predictions based on matrix elements implemented 


\begin{tabular}{|l|c|}
\hline QCD Calculation & $\chi^{2}: x_{g}(10$ bins $)$ \\
\hline LO $m_{b}\left(m_{Z}\right)=3 \mathrm{GeV} / c^{2}$ & 170 \\
NLO $m_{b}\left(m_{Z}\right)=3 \mathrm{GeV} / c^{2}$ & 51 \\
PS $M_{b}=5 \mathrm{GeV} / c^{2}$ & 18 \\
\hline
\end{tabular}

Table 2: $\chi^{2}$ for the comparison of the QCD predictions with the corrected data.

in JETSET which assume massless quarks. The resulting distributions are practically indistinguishable from the massive onesTeven though the large $b$-mass has been seen [19] to affect the $b \bar{b} g$ event rate at the level of $5 \%$. The effect of varying $\alpha_{s}$ within the worldaverage range is similarly small.

We conclude that perturbative QCD in the PS approximation accurately reproduces the gluon distributions in $b \bar{b} g$ events. HoweverTit is interesting to consider the extent to which anomalous chromomagnetic contributions are allowed. The Lagrangian represented by Eq. 1 yields a model that is non-renormalizable. Nevertheless tree-level predictions can be derived [11] and used for a 'straw man' comparison with QCD. For illustration $\Gamma$ the effect of a large anomalous moment $\Gamma \kappa=0.75 \Gamma$ on the shape of the $x_{g}$ distribution is shown in Fig. 3(a); there is a clear depletion of events in the region $x_{g}<0.5$ and a corresponding enhancement for $x_{g} \geq 0.5$. By contrast the shape of the $\cos \theta_{g}$ distribution is relatively unchanged (not shown) $\Gamma$ even by such a large $\kappa$ value. In each bin of the $x_{g}$ distribution $\Gamma$ we parametrised the leading-order effect of an anomalous chromomagnetic moment and added it to the PS calculation to arrive at an effective QCD prediction including the anomalous moment at leading-order. A $\chi^{2}$ minimization fit was performed to the data with $\kappa$ as a free parameter $\Gamma$ yielding $\kappa=-0.011 \pm 0.048$ (stat. $)_{-0.003}^{+0.013}$ (syst.) $\Gamma$ which is consistent with zero within the errors $\Gamma$ with a $\chi^{2}$ of 17.8 for 9 degrees of freedom. The distribution corresponding to this fit is indistinguishable from the PS prediction (Fig. 3(a)) and is not shown. Our result corresponds to $95 \%$ confidence-level (c.1.) upper limits of $-0.11<\kappa<0.08$ (preliminary). 


\section{Conclusion}

In conclusion $\Gamma$ we used the precise SLD tracking system to tag the gluon in 3 -jet $e^{+} e^{-} \rightarrow Z^{0} \rightarrow b \bar{b} g$ events. We studied the structure of these events in terms of the scaled gluon energy and polar angle $\Gamma$ measured across the full kinematic range. We compared our data with perturbative QCD predictions $b$-mass on the shapes of the distributions is small that beyond-LO QCD contributions are needed to describe the energy distribution $\Gamma$ and that the parton shower prediction agrees with the data. We also investigated an anomalous $b$-quark chromomagnetic moment $\Gamma \kappa$ Twhich would affect the shape of the energy distribution. We set preliminary $95 \%$ c.l. limits of $-0.11<\kappa<0.08$.

We thank the personnel of the SLAC accelerator department and the technical staffs of our collaborating institutions for their outstanding efforts on our behalf. We thank A. Brandenburg Г P. Uwer and T. Rizzo for many helpful discussions and for their calculational efforts on our behalf.

This work was supported by Department of Energy contracts: DE-FG02-91ER40676 (BU), DEFG03-91ER40618 (UCSB), DE-FG03-92ER40689 (UCSC), DE-FG03-93ER40788 (CSU), DE-FG0291ER40672 (Colorado), DE-FG02-91ER40677 (Illinois), DE-AC03-76SF00098 (LBL), DE-FG02-92ER40715 (Massachusetts), DE-FC02-94ER40818 (MIT), DE-FG03-96ER40969 (Oregon), DE-AC03-76SF00515 (SLAC), DE-FG05-91ER40627 (Tennessee), DE-FG02-95ER40896 (Wisconsin), DE-FG02-92ER40704 (Yale); National Science Foundation grants: PHY-91-13428 (UCSC), PHY-89-21320 (Columbia), PHY-92-04239 (Cincinnati), PHY-95-10439 (Rutgers), PHY-88-19316 (Vanderbilt), PHY-92-03212 (Washington); the UK Particle Physics and Astronomy Research Council (Brunel, Oxford and RAL); the Istituto Nazionale di Fisica Nucleare of Italy (Bologna, Ferrara, Frascati, Pisa, Padova, Perugia); the Japan-US Cooperative Research Project on High Energy Physics (Nagoya, Tohoku); and the Korea Science and Engineering Foundation (Soongsil). 


\section{References}

[1] See $e g$. S.L. WuTPhys. Rept. 107 (1984) 59.

J. EllisГM. K. GaillardTand G. G. RossTNucl. Phys. B111 (1976) 253; erratum: ibid. B130 (1977) 516.

[2] See eg. P.N. BurrowsTP. OslandГPhys. Lett. B400 (1997) 385.

[3] We do not distinguish between particle and antiparticle.

[4] C. J. S. Damerell et al.TNucl. Inst. Meth. A288 (1990) 236.

[5] K. Abe et al.rNucl. Inst. Meth. A400 (1997) 287.

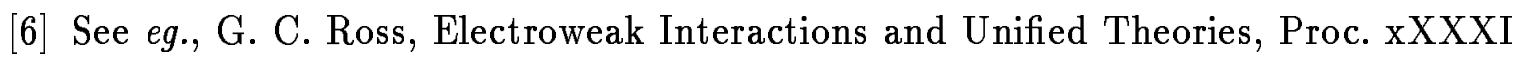
Rencontre de Moriond $\Gamma$ 16-23 March 1996Г Les Arcs $\Gamma$ Savoie $\Gamma$ France $\Gamma$ Editions Frontieres (1996)Гed. J. Tran Thanh VanTp 481.

[7] SLD Collab.TK. Abe et al.ГPhys. Rev. D59 (1999) 012002.

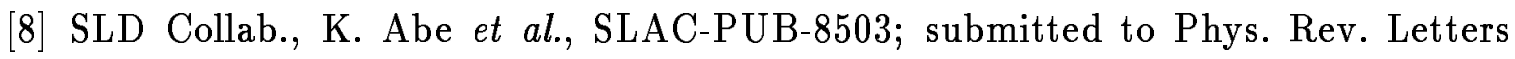
and contributed to this conference.

[9] SLD Collab.TK. Abe et al.TPhys. Rev. D60 (1999) 92002.

[10] W. BernreutherГA. BrandenburgГР. UwerГPhys. Rev. Lett. 79 (1997) 189.

A. Brandenburg TP. UwerTNucl. Phys. B515 (1998) 279.

[11] T. RizzoTPhys. Rev. D50 (1994) 4478Гand private communications.

[12] SLD Design ReportTSLAC Report 273 (1984).

[13] P.J. DervanTBrunel Univ. Ph.D. thesis; SLAC-Report-523 (1998).

[14] JADE Collab.TW. Bartel et. al.ГZ. Phys. C33 (1986) 23.

[15] T. SjöstrandГComp. Phys. Commun. 82 (1994) 74.

[16] P. N. BurrowsTZ. Phys. C41 (1988) 375.

OPAL Collab.TM. Z. Akrawy el al.Гibid. C47 (1990) 505. 
[17] SLD Collab.ГK. Abe et al.ГPhys. Rev. Lett. 79 (1997) 590.

[18] We expect less than $0.4 \%$ of the selected sample to comprise events of the type $\mathrm{e}^{+} \mathbf{e}^{-} \rightarrow q \bar{q} g \Gamma$ with $g \rightarrow b \bar{b}$. In the evaluation of the purity only true $b \bar{b} b \bar{b}$ events were considered as signal $b \bar{b} g$ events; $q \bar{q} b \bar{b}$ events $(q \neq b)$ were considered as backgrounds.

[19] DELPHI Collab.ГP. Abreu et al.ГPhys. Lett. B418 (1998) 430;

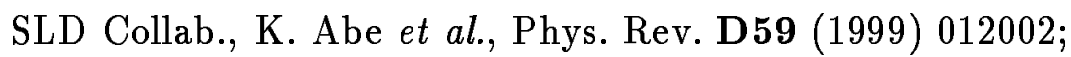

A. Brandenburg et al.ГPhys. Lett. B468 (1999) 168;

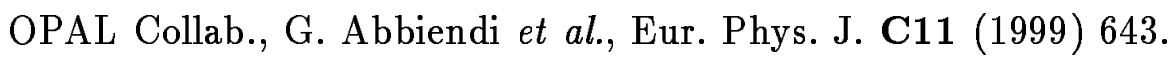

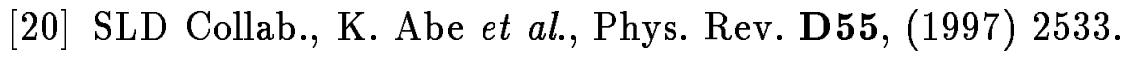

\section{${ }^{* *}$ List of Authors}

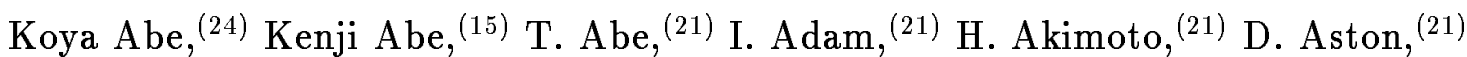
K.G. Baird $\mathbb{T}^{(11)}$ C. Baltay $\mathbb{T}^{(30)}$ H.R. Band $\mathbb{T}^{(29)}$ T.L. Barklow $\mathrm{I}^{(21)}$ J.M. Bauer $\mathrm{T}^{(12)}$ G. Bellodit ${ }^{(17)}$ R. Berger $T^{(21)}$ G. Blaylock $T^{(11)}$ J.R. Bogart $T^{(21)}$ G.R. Bower $T^{(21)}$

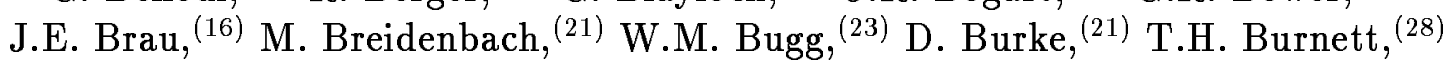
P.N. Burrows $\mathrm{I}^{(17)}$ A. Calcaterra $\mathrm{I}^{(8)}$ R. Cassell( ${ }^{(21)}$ A. ChouI ${ }^{(21)}$ H.O. CohnI ${ }^{(23)}$ J.A. Coller $T^{(4)}$ M.R. Convery $T^{(21)}$ V. Cook $T^{(28)}$ R.F. Cowant $T^{(13)}$ G. Crawford $T^{(21)}$ C.J.S. Damerelli ${ }^{(19)}$ M. Daoudi $T^{(21)}$ S. DasuT $T^{(29)}$ N. de Groot $\mathbb{T}^{(2)}$ R. de Sangrot ${ }^{(8)}$ D.N. Dong $\mathrm{T}^{(13)}$ M. Doser $\mathrm{T}^{(21)}$ R. DuboisTI. Erofeeva $\mathrm{T}^{(14)}$ V. Eschenburg $\mathrm{T}^{(12)}$

E. Etzion $\mathrm{T}^{(29)}$ S. Fahey $\mathrm{T}^{(5)}$ D. Falciai ${ }^{(8)}$ J.P. Fernandez $\mathrm{T}^{(26)}$ K. Flood $\mathrm{T}^{(11)}$ R. Frey $\mathrm{T}^{(16)}$

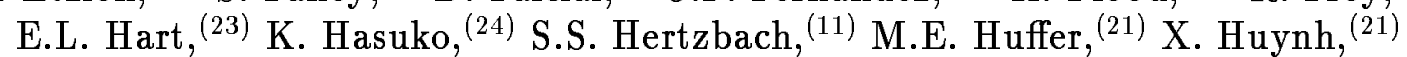

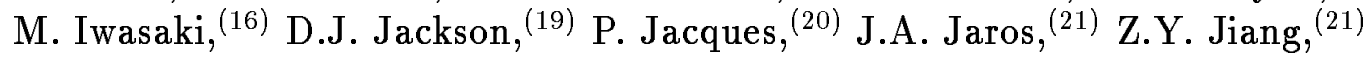
A.S. Johnson $T^{(21)}$ J.R. Johnson $T^{(29)}$ R. Kajikawa $T^{(15)}$ M. Kalelkar $T^{(20)}$ H.J. Kang $T^{(20)}$

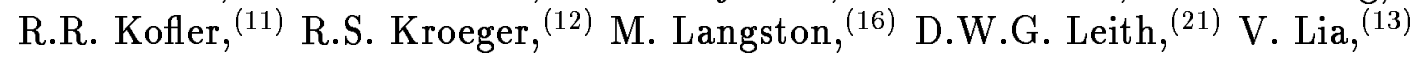
C. Lin $\Gamma^{(11)}$ G. Mancinelli $\mathrm{T}^{(20)}$ S. Manly $\mathrm{T}^{(30)}$ G. Mantovani $\mathrm{T}^{(18)}$ T.W. Markiewicz $\mathrm{T}^{21)}$

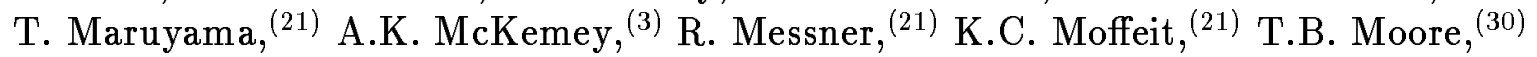
M. Morii $\mathbb{T}^{(21)}$ D. Muller $T^{(21)}$ V. Murzin $\mathbb{T}^{(14)}$ S. Narita $\mathrm{I}^{(24)}$ U. Nauenberg $\mathrm{I}^{(5)}$ H. NealI ${ }^{(30)}$

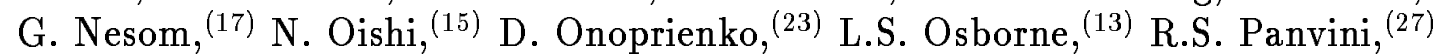

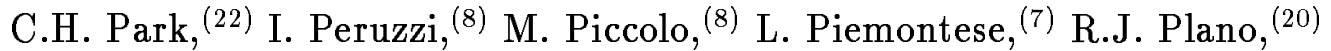
R. Prepost ${ }^{(29)}$ C.Y. Prescott $\mathrm{I}^{(21)}$ B.N. Ratcliff( ${ }^{(21)}$ J. Reidy $\mathrm{I}^{(12)}$ P.L. Reinertsen $\mathrm{I}^{(26)}$ L.S. Rochester $T^{(21)}$ P.C. Rowson $T^{(21)}$ J.J. Russelli ${ }^{(21)}$ O.H. Saxtont ${ }^{(21)}$ T. Schalk $\mathbb{T}^{(26)}$ B.A. Schumm $T^{(26)}$ J. Schwiening $\mathrm{I}^{(21)}$ V.V.Serbo $\mathrm{I}^{(21)}$ G. Shapiro $\mathrm{I}^{(10)}$ N.B. Sinev $\mathrm{I}^{(16)}$

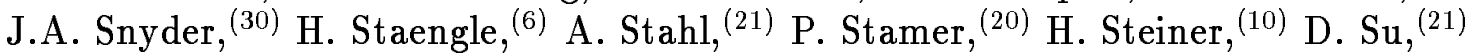

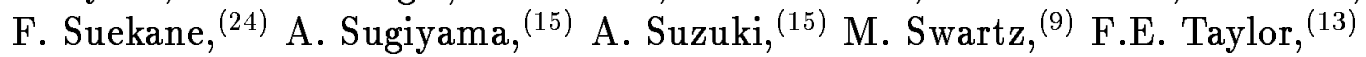
J. Thom $T^{(21)}$ E. Torrence $I^{(13)}$ T. Usher $\Gamma^{(21)}$ J. Va'vra $\Gamma^{(21)}$ R. Verdier $\left(^{(13)}\right.$

D.L. Wagner $T^{(5)}$ A.P. Waite $I^{(21)}$ S. Walston $T^{(16)}$ A.W. Weidemann $T^{(23)}$ E.R. Weiss $I^{(28)}$ 
J.S. Whitaker $\mathrm{T}^{(4)}$ S.H. Williams $\mathrm{I}^{(21)}$ S. Willocq $\mathrm{T}^{(11)}$ R.J. Wilson $\mathrm{I}^{(6)}$

W.J. Wisniewski $\mathrm{I}^{(21)}$ J.L. Wittlin $\mathrm{I}^{(11)}$ M. Woods $\mathrm{I}^{(21)}$ T.R. Wright $\mathrm{I}^{(29)}$

R.K. Yamamoto ${ }^{(13)}$ J. Yashima $\mathrm{I}^{(24)}$ S.J. Yellin $\mathrm{I}^{(25)}$ C.C. Young $\mathrm{I}^{(21)}$ H. Yuta. ${ }^{(1)}$

(1) Aomori University, Aomori, 030 Japan,

(2) University of Bristol, Bristol, United Kingdom,

${ }^{(3)}$ Brunel University, Uxbridge, Middlesex, UB8 3PH United Kingdom,

${ }^{(4)}$ Boston University, Boston, Massachusetts 02215,

(5) University of Colorado, Boulder, Colorado 80309,

${ }^{(6)}$ Colorado State University, Ft. Collins, Colorado 80523,

${ }^{(7)}$ INFN Sezione di Ferrara and Universita di Ferrara, I-44100 Ferrara, Italy,

${ }^{(8)}$ INFN Laboratori Nazionali di Frascati, I-00044 Frascati, Italy,

(9) Johns Hopkins University, Baltimore, Maryland 21218-2686,

${ }^{(10)}$ Lawrence Berkeley Laboratory, University of California, Berkeley, California 94720,

(11) University of Massachusetts, Amherst, Massachusetts 01003,

${ }^{12)}$ University of Mississippi, University, Mississippi 38677,

${ }^{(13)}$ Massachusetts Institute of Technology, Cambridge, Massachusetts 02139,

${ }^{(14)}$ Institute of Nuclear Physics, Moscow State University, 119899 Moscow, Russia,

${ }^{(15)}$ Nagoya University, Chikusa-ku, Nagoya, 464 Japan,

(16) University of Oregon, Eugene, Oregon 97403,

(17) Oxford University, Oxford, OX1 3RH, United Kingdom,

(18) INFN Sezione di Perugia and Universita di Perugia, I-06100 Perugia, Italy,

${ }^{(19)}$ Rutherford Appleton Laboratory, Chilton, Didcot, Oxon OX11 OQX United Kingdom,

${ }^{(20)}$ Rutgers University, Piscataway, New Jersey 08855,

${ }^{(21)}$ Stanford Linear Accelerator Center, Stanford University, Stanford, California 94309 ,

${ }^{(22)}$ Soongsil University, Seoul, Korea 156-7433,

(23) University of Tennessee, Knoxville, Tennessee 37996,

(24) Tohoku University, Sendai, 980 Japan,

${ }^{(25)}$ University of California at Santa Barbara, Santa Barbara, California 93106,

${ }^{(26)}$ University of California at Santa Cruz, Santa Cruz, California 95064,

${ }^{(27)}$ Vanderbilt University, Nashville, Tennessee 37235,

(28) University of Washington, Seattle, Washington 98105,

${ }^{(29)}$ University of Wisconsin, Madison, Wisconsin 53706,

${ }^{(30)}$ Yale University, New Haven, Connecticut 06511. 